NOTICIAS Y OPINIONES

\title{
Amblyrhynchus cristatus, el único lagarto marino del mundo.
}

\author{
Amblyrhynchus cristatus, the only sea lizard in the world. \\ Odalys Torres
}

DOI. $10.21931 / \mathrm{RB} / 2017.02 .02 .10$

\section{RESUMEN}

\begin{abstract}
Amblyrhynchus cristatus perteneciente a la familia Iguanidae es comúnmente conocida como iguana marina. Se establece en la región insular del país dentro del Parque Nacional Galápagos. Su peculiar capacidad de alimentarse y llevar una buena parte de su vida bajo el agua, es lo que la convierte en una especie única. Los estudios realizados demuestran que esta especie sufrió importantes adaptaciones en su cuerpo que le permiten soportar los cambios de temperatura entre el ambiente y el medio acuático. La Amblyrhynchus cristatus. se ve amenazada por la incorporación de especies introducidas al ecosistema de las islas, se ha propuesto varias alternativas para poder impulsar la conservación tanto de las especies endémicas como de su hábitat.
\end{abstract}

Palabras Claves: Amblyrhynchus cristatus, iguana marina, Galápagos, temperatura, hábitat, interacciones.

\section{ABSTRACT}

Amblyrhynchus cristatus belonging to the family Iguanidae is commonly known as marine iguana. It is established in the insular region of the country within the Galapagos National Park. Her peculiar ability to feed herself and live a good part of her life under water, is what makes her a unique species. Studies show that this species underwent important adaptations in its body that allow it to withstand the temperature changes between the surface and aquatic environment. The Amblyrhynchus cristatus is threatened by the incorporation of introduced species into the ecosystem of the islands, several alternatives have been proposed to promote the conservation of endemic species and their habitat.

Key Words: Amblyrhynchus cristatus, marine iguana, Galapagos, temperature, habitat, interactions

\section{Introducción}

Amblyrhynchus cristatus, conocida comúnmente como iguana marina es una especie endémica de la región insular de la Republica de Ecuador, representa una figura importante dentro del desarrollo del hábitat que existen en el Archipiélago de Galápagos (Figura 1). E habitante más característico de las costas rocosas de las Galápagos es indudablemente la iguana marina, único lagarto marino viviente en el mundo. Sin duda esta especie de reptiles forma parte del grupo de vertebrados que domina la escena en las islas, cuyas características logradas por esta especie solamente las pudo obtener en este ecosistema inusualmente árido para una latitud tropical, ya que poseen un clima diferente en cada una de las islas e islotes que lo conforman'1.

Este articulo busca recopilar y presentar información referente a la morfología fisiología y la correlación con el ecosistema en el que se desarrolla la Amblyrhynchus cristatus, además de las amenazas que sufre esta especie en relación a la acción humana y especies introducidas dentro de su ecosistema.

\section{Hábitat de la Amblyrhynchus cristatus: Parque Nacional Galápagos}

Actualmente las islas Galápagos constituyen una provincia de 12.000 habitantes aproximadamente, se convirtió en la sede de la Estación Científica Charles
Darwin, el manejo de esta zona se remonta al año 1936 con el primer Plan de Manejo del área que comprende un total de $7.995 \mathrm{~km}^{2}$, cabe recalcar que el 3\% de este parque está destinado al uso y usufructo de los asentamientos humanos ${ }^{2}$. Separado de las costas ecuatorianas por $1000 \mathrm{~km}$, este archipiélago formado por islas oceánicas de origen volcánico presenta dos estaciones bien marcadas: cálida y lluviosa, esta última permite el desarrollo de comunidades vegetales diferentes en cada una de las islas, la presencia de precipitaciones de 300 a $1700 \mathrm{~mm}$ anuales ${ }^{3}$, acompañadas de un descenso de las temperaturas. El Parque Nacional Galápagos está incluido en un archipiélago de 121 islas, islotes y rocas (Snell et al. 1995; Anexo 2), dispersos en 38.000 $\mathrm{km}^{2}$ de mar interior (Plan de Manejo de la Reserva de Recursos Marinos de Galápagos, 1992), presentando un elevado endemismo y otras características biológicas y físicas únicas. Actualmente las Islas Galápagos son una de las zonas de Ecuador con su flora y fauna mejor conservada. Según La Lista Roja la población de iguanas marinas en las islas es difícil de calcular con certeza más se conoce poblaciones que van desde las 4,000 hasta las 30,000 dependiendo de la isla en la que desarrollaron su hábitat. Además de la Amblyrhynchus cristatus existe una gran cantidad de especies endémicas del PNG (Parque Nacional Galápagos) y la RNG (Reserva Nacional Galápagos), se reposta la existencia de 2,900 especies de organismos marinos, de los cuales

${ }^{1}$ Universidad de Investigación de Tecnología Experimental Yachay Tech, Urcuqui, Ecuador.

Correspondencia: karen.torres@yachaytech.edu.ec 


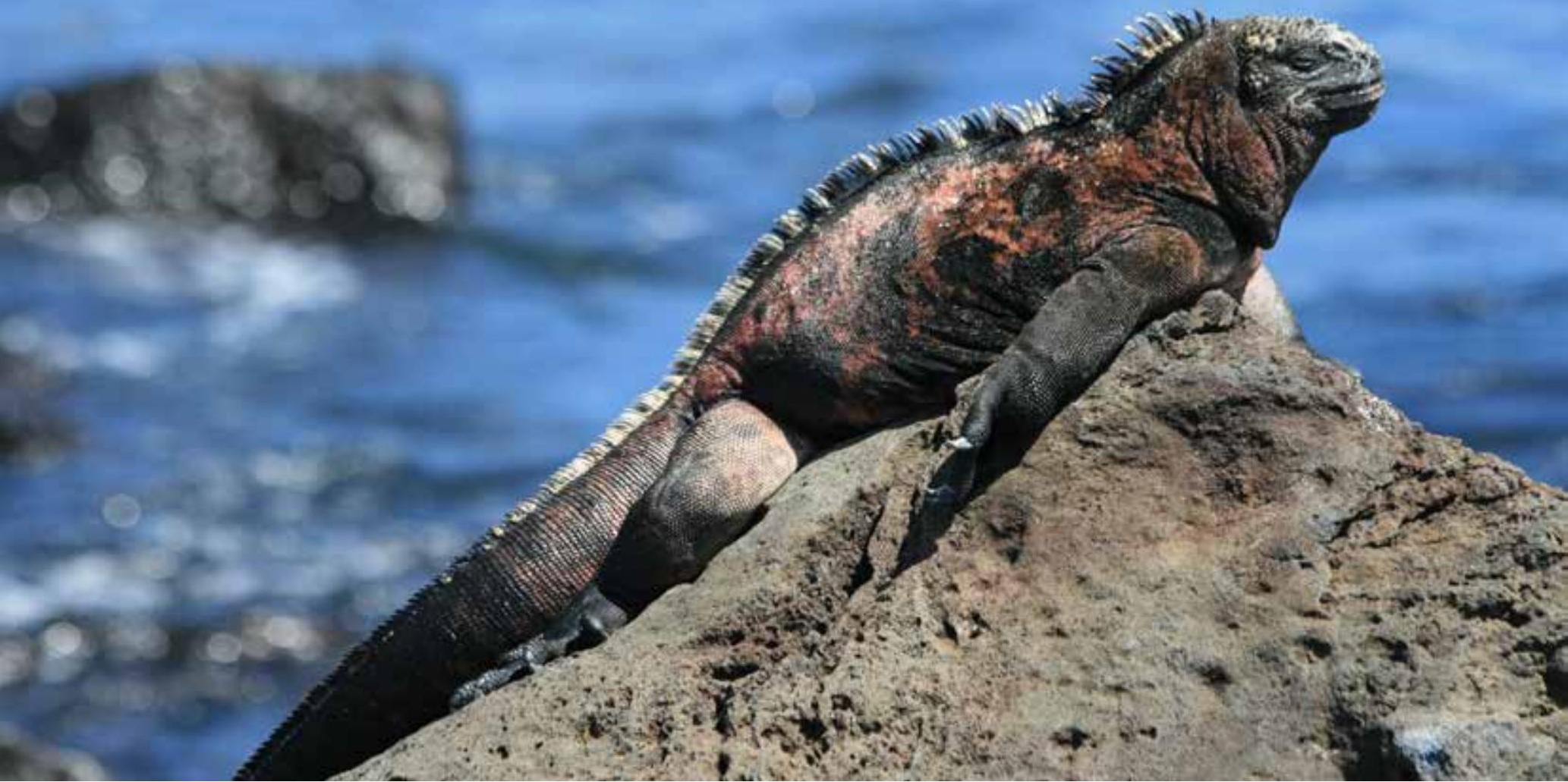

Figura 1: Proceso de termoregulación en las costas del Amblyrhynchus cristatus.

encontramos $18,2 \%$ de endemismo, además de un promedio por grupo biótico de endemismo del $25 \%{ }^{4}$. Entre las especies que se pueden encontrar en PNG se encuentran: Grapsus grapsus, Pelecanus occidentalis, Cheloniidae (Chelonoidis nigra), Testundinidae, Phalacrocoracidae, Spheniscidae, Diamedeidae. Carcharhinidae (Carcharhinus galapagensis). Dado que el Parque Nacional Galápagos posee una gran cantidad de especies endémica ha sido considerada como uno de los lugares con mayor importancia biológica en nuestro país.

El clima tropical de esta región insular genera un ambiente de desarrollo de especies capaces de adaptarse a las zonas áridas y semi-humedas generadas a partir de un fenómeno frecuente de las zonas altas conocido como garúa, este término hace alusión a precipitaciones de baja intensidad. Al presentarse una gradiente de clima se puede observar zonación vegetal que comprende desde manglares hasta arbustos tolerantes de condiciones salinas, además de la presencia de helechos, juncias y hierbas ${ }^{1}$.

El aislamiento que se evidencia en las islas permite el desarrollo de características especiales en cada una de las especies que forman parte del parque, han generado una gran cantidad de adaptaciones propias del ecosistema único que se desarrolla en cada isla. Después de una evaluación de taxonomías intraespecífica se pudo determinar 7 diferentes subespecies de iguanas marinas dentro de las islas: Amblyrhynchus cristatus ssp. albemarlensis ${ }^{5}$ ssp. cristatus, ssp. hassi, ssp. mertensi, ssp. nanus, ssp. sielmanni, ssp. Venustissimus. Los efectos del Fenomeno del Niño generan un gran declive en la población de esta especie, se ha calculado una disminución del $85 \%$ de la población cada ocasión en la que este se ha presentado. Además, la población se ve afectada por la contaminación y especies introducidas que se han convertido en predadores ${ }^{6}$.

\section{Amblyrhynchus cristatus: morfología y fisiología}

Amblyrhynchus cristatus proveniente de la familia iguanidae, es un reptil marino endémico de las Islas Galápagos con 10 subpoblaciones se estima que ocupan un total de $500 \mathrm{~km}^{2}$. Amblyrhynchus cristatus es la única iguana marina por lo que viven en las zonas rocosas de Galápagos cercanas a las costas. Son un grupo de vertebrados amniotas provistos de escamas epidérmi- cas de queratina. Además, poseen una circulación incompleta y glóbulos rojos nucleados? ${ }^{7}$ Una de sus características más importantes es su capacidad de permanecer bajo el mar por hasta 45 minutos. Poseen un particular hocico de punta lo cual les facilita la extracción de algas de las profundidades gracias a sus dientes afilados. Su nado se basa en una ondulación lateral de sus cuerpos, tienen colas aplanadas, garras largas y afiladas a diferencia de sus primos terrestres, esta característica les facilita la sujeción a las rocas localizadas a orillas del mar (Figura 2). Suelen en ocasiones tomar coloraciones rojizas y verdosas. Su piel dura impide la desecación y en su mayoría es impermeable al igual que la cascara de sus huevos.

Esta especie presenta un característico color negro que lo hace muy evidente en la arena de la playa pero contribuye en su camuflaje en las áreas rocosas además de permitirles una mejor absorción del calor proveniente del sol. (Fig.1) Una de las características importantes de este reptil es su sangre fría y necesitan mantener su calor corporal, por lo que suelen mantenerse juntas, comportamiento que no es común en la mayoría de reptiles?.

Las adaptaciones de estos animales les permiten diferenciarse de las iguanas terrestres (Conolophus subcristatus), su coloración café y su comportamiento especialmente esquivo caracteriza a los miembros de esta especie, completamente diferente a la $\mathrm{Am}$ blyrhynchus cristatus. Pueden llegar a alcanzar un peso de 0,5 a $1,5 \mathrm{~kg}$, lo cual evidencia un eficiente desarrollo de su morfología que les ha permitido tener acceso a las zonas en las que habitan y a los alimentos que forman parte de su dieta actual.

La Amblyrhynchus cristatus sigue el sistema regular de apareamiento de los reptiles que consiste principalmente en la búsqueda de pareja por parte de los machos, y en la disponibilidad espacial y temporal de las hembras ${ }^{10}$. Generalmente los individuos machos tienden a aparearse con varios individuos del sexo contrario durante la estación ${ }^{11}$. La monogamia en esta especie en poco frecuente. En consecuencia, la duración del emparejamiento varía mucho entre individuos de la misma especie el cual en la mayoría de los casos dura muy poco. La incubación de los huevos va desde los 86 a 120 días, mientras que la hembra se encarga del nido únicamente durante los primeros 16, estos huevos se depositan una zona específica denominada lugar de excavación. 


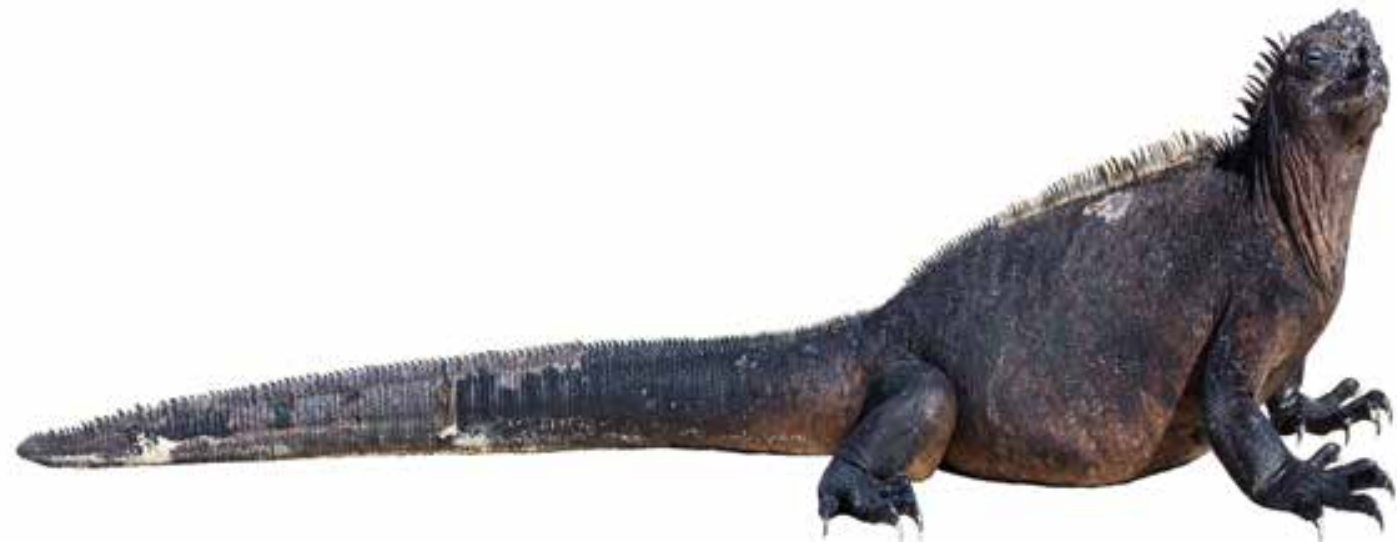

Figura 2: Morfología del Amblyrhynchus cristatus vista lateral.

Si bien es cierto no existen registros de una enfermedad que afecte a esta especie en específico, se han reportado casos de muerte de iguanas por un desajuste nutricional. Sin embargo, al ser reptiles de la familia Iguanidae podrán ser propensas a compartir patologías tales como: osteoporosis, osteodistrofia, infecciones respiratorias producto de la exposición prolongadas a temperaturas muy bajas ${ }^{12}$.

\section{Interacción de la Amblyrhynchus cristatus con el ecosistema de las islas.}

Como ya se mencionó anteriormente dentro de las islas existen 7 diferentes subespecies de la Amblyrhynchus cristatus todo esto debido a las adaptaciones que desarrolla cada subespecie en función del ambiente en el que se desarrolla. Estas iguanas pueden llegar a vivir desde 5 a 12 años en su medio natural y durante ese tiempo contribuir al balance natural establecido en las islas por las especies endémicas que se han encontrado ahí desde los tiempos de Darwin. Estos animales duermen expuestos a las costas y en los árboles de mangle que rodean las playas. Los zoólogos especulan que la razón de las adaptaciones de esta peculiar iguana a la comida marina es debido a la escasez de comida en el territorio insular, al ser islas de origen volcánico la vegetación es escasa e inclusive en algunos casos inexistente. (Figua 3)

\section{Amenazas directas al ecosistema y a la especie}

La introducción de mamíferos como los perros y gatos ferales provoca la depredación y disminución de las poblaciones de esta especie endémica tanto de jóvenes como de adultos. Se han obtenido alarmantes cifras que nos muestran que la mayoría de individuos de la especie no logra llegar a la edad reproductiva. En algunas islas los gatos victimaban a gran escala a las iguanas marinas. Su investigación era en su mayor parte en la zona costera del Archipiélago. El estado actual de las islas y la disminución de las poblaciones se debe además a la gran afectación que tiene la corriente del Niño en las poblaciones de especies costeras tal como la Amblyrhynchus cristatus ${ }^{11}$.

Presumiblemente un programa de monitoreo de eclosión y la reducción del número de mamíferos y demás especies introducidas sería la solución ideal al problema al que se enfrenta esta especie. (Figura 4)

\section{CONCLUSIONES}

La especie Amblyrhynchus cristatus es una de las principales criaturas que forman parte de los ecosistemas de las islas de for-

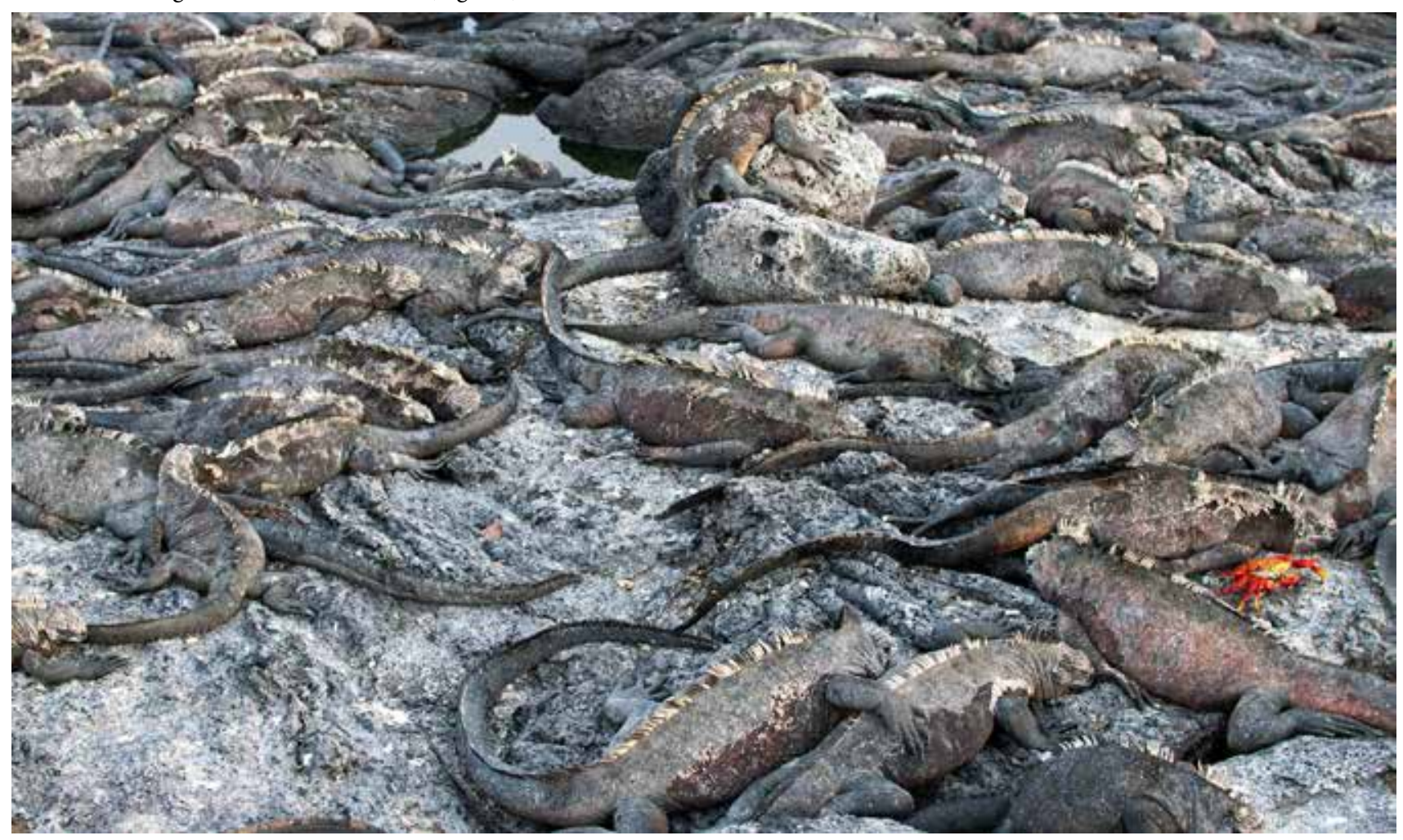

Figura 3: Comportamiento caracteristico de reptiles, se agrupan para conservar calor. 


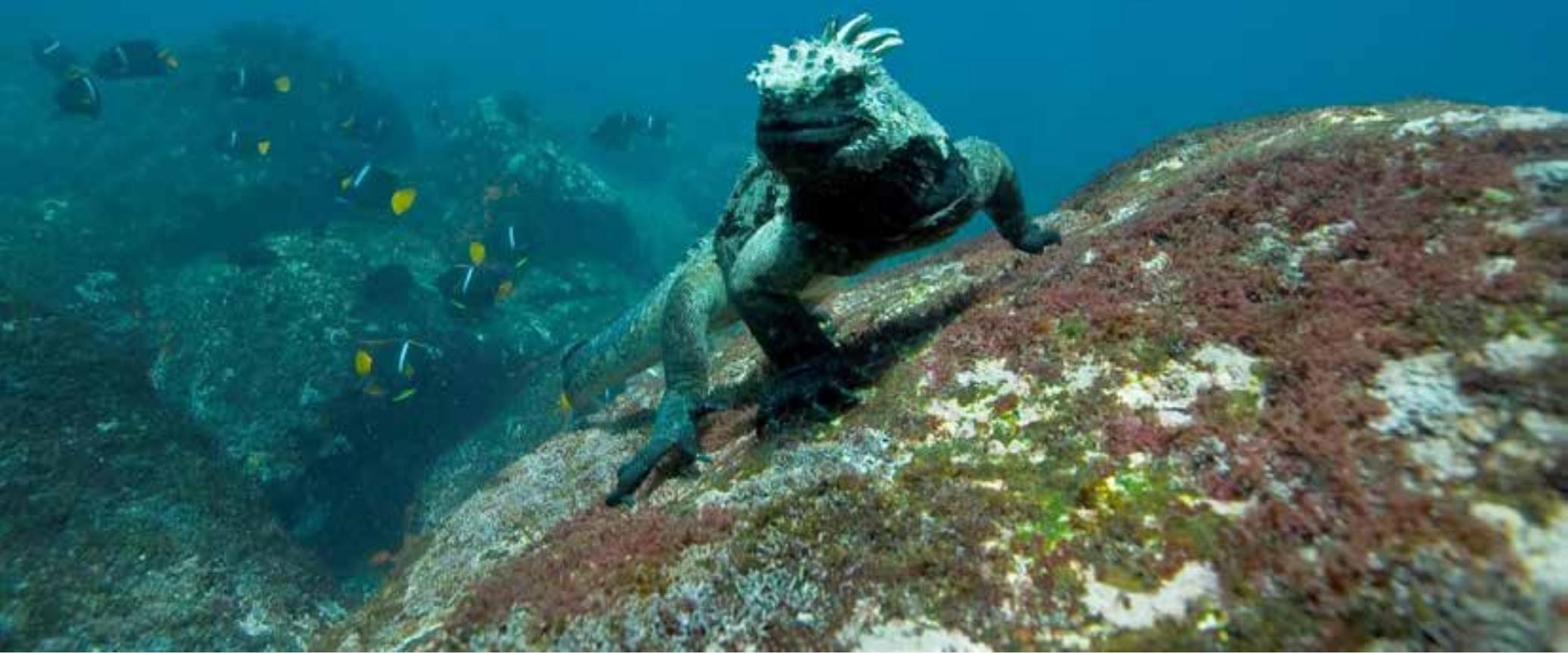

Figura 4: Proceso de alimentacion del Amblyrhynchus cristatus de algas provenientes de los lechos marinos.

mación volcánica conocidas como el Archipiélago de Galápagos, estas son un icono de especies endémicas, dadas las adaptaciones que las especies sufren debido a las condiciones extremas a las que se someten, la especie estudiada logró desarrollar siete subespecies debido a este factor especie-ecosistema propio de cada formación insular. Forman parte importante del ecosistema que les rodea porque al ser un ambiente aislado se ha desarrollado un ecosistema único de especies endémicas que se busca no perturbar en la medida de lo posible. La Amblyrhynchus cristatus presenta boca puntiaguda, dientes y garras afilados y la capacidad de permanecer bajo el agua durante largos períodos de tiempo, su comportamiento pacífico y todas sus demás características convierten a esta especie en única he irremplazable en las costas insulares de La Reserva Nacional Galápagos. Todas las especies perecientes tanto a la reserva como al Parque Nacional Galápagos se han convertido en víctima de las especies introducidas. La implementación de planes de control y disminución del factor introducido solucionarían los grandes problemas de disminución de población de la especie, además de más control de las actividades humanas en la zona, con el objetivo de concientizar a la gente y controlar que las actividades que estos realizan para su usufructo afecten de la menor manera a los ecosistemas propios de las islas.

\section{Referencias bibliográficas}

1. Departamento Científico de Entomología, Museo de La Plata; investigadora Independiente del CONICET. (2011). Las Islas Galápagos: Un Paraíso Amenazado. Revista Museo. Recuperado de http://sedici.unlp.edu.ar/bitstream/handle/10915/48635/Documento_completo.pdf?sequence $=1$

2. Parque Nacional Reserva Marina de Galápagos Ecuador. 1996. PLAN DE MANEJO DEL PARQUE NACIONAL GALÁPAGOS. Servicio Parque Nacional Galápagos Instituto Ecuatoriano Forestal y de Áreas Naturales y Vida Silvestre. Recuperado de http://www. miliarium.com/paginas/leyes/internacional/Ecuador/Conservacion/PNG_pm96_es.pdf
3. Solís, M. A. 1979. Galápagos y su naturaleza. Geografía, Ecología y Conservación. Biblioteca Ecuador. Quito.

4. Lisseth Encalada Serrano. 2013. Ecosistemas de las Islas Galápagos Universidad de Cuenca. Recuperado de http://es.slideshare.net/ LissethEncaladaSerrano/ecosistemas-de-las-islas-galpagos

5. Nelson, K., Snell, H. \& Wikelski, M. 2004. Amblyrhynchus cristatus ssp. albemarlensis. The IUCN Red List of Threatened Species 2004: e.T44173A10867894. http://dx.doi.org/10.2305/IUCN.UK.2004. RLTS.T44173A10867894.en. Descargado el 01 de Febrero del 2017.

6. The IUCN Red List of Threatened Species: Amblyrhynchus cristatus - published in 2004. http://dx.doi.org/10.2305/IUCN.UK.2004. RLTS.T1086A3222951.en

7. Agustín Alvear. 2013. Las Iguanas Marinas Recuperado de https://prezi. com/wnxtwlnah3ao/las-iguanas-marinas/

8. Galapagos Islands Tour Guide. 2003-2016. Iguana Marina de Galápagos Recuperado de http://www.galapagos-islands-tourguide. com/iguana-marina.html

9. Ismael Pérez Fernández. 2011. Lagartos, iguanas, tortugas y la evolución abofeteándote la cara. Recuperado de http://naukas. com/2011/08/10/lagartos-iguanas-tortugas-y-la-evolucion-abofeteandote-la-cara/

10. POUGH, F.H., ANDREWS, R.M., CADLE, J.E., CRUMP, M.L., SAVÍTZKY, A.H. \& WELLS, K.D. (2001): Herpetología. 2nd ed., New Jersey. Prentice Hall.

11. Linda J. Cayot, Kornelia Rassmann y Fritz TriUmich. (1994): ¿ESTAN LAS IGUANAS MARINAS AMENAZADAS EN ISLAS CON DEPREDADORES INTRODUCIDOS? Noticias de Galapagos v.51, 52, 53 Versión en Español

12. Cecilia Alvear. (2013): Las Autoridades de Galapagos Investigan Muerte de Iguanas Marinas en Tortuga Bay. Recuperado de http://www.galapagosdigital.com/espanol/2013/09/13/las-autoridades-de-galapagos-investigan-muerte-de-iguanas-marinas-en-tortuga-bay/

Recibido: Enero de 2017. Aprobado: Abril de 2017. 


\section{Gestione sus autores}

¿QUÉ ES

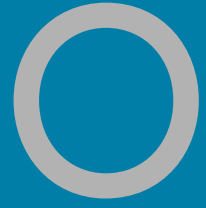

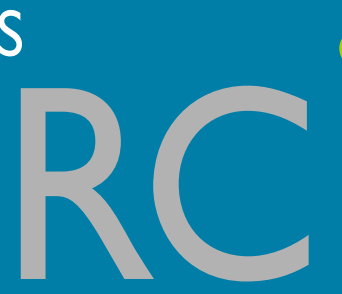

0

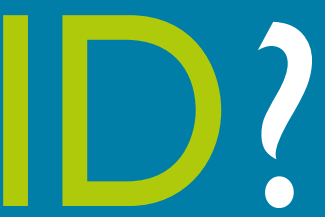

ORCID puede ayudarle a gestionar mejor sus autores $\mathrm{y}$ revisores,

proporcionando un registro único de identificadores permanentes

para investigadores y académicos, que es abierto, no propietario,

transparente, móvil y basado en la comunidad.

Todo lo que hacemos está impulsado por los datos, así que necesitamos identificadores de autor fiables. Pero no puede haber identificadores de autor fiables $\sin$ ORCID.

Paul Peters, director de estrategia en Hindawi Publishing

El sistema ORCID no sólo permite mejorar los procesos

de publicación, sino que

la participación de los editores también permite la mejora de los procesos para socios como los organismos de financiación y las universidades.

Craig Van Dyck, Vicepresidente de Gestión de Contenidos de Global, Global Research, Wiley

Como organización independiente y sin ánimo de lucro diseñada para beneficiar a un amplio rango de organizaciones, ORCID es única en su capacidad para atravesar disciplinas, sectores de investigación y fronteras nacionales. La capacidad de conectar la investigación y los investigadores enriquece el proceso del descubrimiento científico y mejora la eficiencia de la financiación y la colaboración científicas. ORCID está trabajando con todos los sectores de la comunidad científica para hacer realidad esta visión.
¿Cómo podemos agilizar nuestro proceso de presentación de manuscritos? ¿Podemos reducir el coste de gestionar y eliminar la ambigüedad de nuestras bases de datos de autores y revisores? ¿Cómo podemos mejorar la exactitud de nuestra búsqueda por autor? ¿Por qué tenemos tantos duplicados en los registros de autor? ¿Dónde encontramos los revisores más cualificados?

Las editoriales académicas y de investigación se enfrentan diariamente a problemas de autoría. Usted se esfuerza por mantener la precisión en las bases de datos de autores, que son necesarias para entender la historia de un autor, para realizar búsquedas basadas en nombres precisos, y para encontrar y gestionar los revisores. En ausencia de identificadores de autor únicos, a menudo usted se ve obligado a eliminar manualmente la ambigüedad de los metadatos para que coincidan correctamente los autores con sus artículos.

Mediante la vinculación de los investigadores y profesores con sus publicaciones, ORCID sirve como un aglutinador de información, y permite a los investigadores y editores gestionar de manera más eficiente y eficaz la difusión de resultados de investigación. ORCID le permite enlazar de forma fiable y sin ambigüedad, los autores con su producción académica completa, correcta y actual, que le ayuda a controlar sus contribuciones únicas.

\section{ORCID ayuda a los editores:}

- Identificar y distinguir claramente a los autores.

- Simplificar los procesos de entrega de manuscritos.

- Encontrar rápidamente lectores y colaboradores.

- Mejorar la velocidad y precisión de búsqueda por autor.

- Mantener la conformidad con el depósito obligatorio de los resultados y los artículos de investigación.

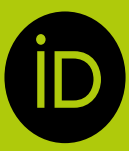
Visite Orcid. Oró para saber más

Únase a ORCID hoy y pase a formar parte de la solución. 